\title{
Diversité Et Importance Socio-Economique Des Espèces Fruitières Sauvages Comestibles En Zone Kasa (Sud-Ouest Du Sénégal)
}

\author{
Yves Djihounouck, Doctorant \\ Laboratoire de Botanique et Biodiversité, \\ Département de Biologie Végétale, Faculté des Sciences et Techniques, \\ Université Cheikh Anta Diop de Dakar, Sénégal \\ Doudou Diop, Docteur \\ Laboratoire de Botanique, Institut Fondamentale d'Afrique Noire (IFAN), \\ Université Cheikh Anta Diop de Dakar, Sénégal \\ Sara Danièle Dieng, Docteur \\ Institut des Sciences de l'Environnement, Facultés des Sciences et \\ Techniques, Université Cheikh Anta Diop de Dakar, Sénégal \\ Seyni Sane, Docteur \\ Laboratoire de Botanique et Biodiversité, \\ Département de Biologie Végétale, Faculté des Sciences et Techniques, \\ Université Cheikh Anta Diop de Dakar, Sénégal \\ César Bassène, Docteur \\ Section Productions Végétales et Agronomie, UFR des Sciences \\ Agronomiques, de l'Aquaculture et des Technologies Alimentaires, \\ Université Gaston Berger de Saint Louis, BP \\ Mame Samba Mbaye, Professeur \\ Kandioura Noba, Professeur \\ Laboratoire de Botanique et Biodiversité, \\ Département de Biologie Végétale, Faculté des Sciences et Techniques, \\ Université Cheikh Anta Diop de Dakar, Sénégal
}

Doi: 10.19044/esj.2018.v14n36p352 URL:http://dx.doi.org/10.19044/esj.2018.v14n36p352

Abstract
Wild fruit species provide local people with subsistence products and
cash income. In addition to their food value, different used organs can,
eventually, compromise the availability of these resources. This study aims
to identify edible woody species in Kasa Zone and to analyze their socio-
economic importance. An ethnobotanical survey was conducted with 82
informants. Data collected were analyzed using Epi Info software and uses of
species were classified into six categories. A Factorial Correspondence 
Analysis (FCA) highlighted species-organ relationships. Frequency of citations, fidelity index, selling price were used to show the socio-economic importance of the species. Results identified 62 edible species belonging to 31 families and 54 genera. These species were dominated by Annonaceae, Apocynaceae and generic diversity was more pronounced among Anacardiaceae. Identified species were mainly used for food (53\%), energy (16\%), technology (12\%) and medicinal purposes (12\%). The FCA showed three organ-use groups with a strong correlation between fruit and root on the one hand and leaf, bark and wood on the other. The study also identified 16 commercialized species. The fruit was the main organ consumed and marketed. The highest number of organs commercialized were recorded for Elaeis guineensis (4 organs) and Borassus aethiopium (3). These products, often collected and sold by women and children, came from villages and transited to markets through the "bana bana". Their prices depended on the locality isolation and the production period. These results open up perspectives for better management of the supply and commercialization of these forest resources.

Keywords: Edible woody species, organs, marketing, Kasa Zone, Senegal

\section{Resume}

Les fruitiers sauvages fournissent aux autochtones des produits de subsistance et des revenus. Cette étude a pour objectif d'identifier les espèces comestibles de la zone Kasa et d'analyser leur importance socio-économique. Une enquête ethnobotanique a été menée auprès de 82 informateurs. Les données collectées ont été dépouillées avec le logiciel Epi Info et les utilisations classées en six catégories. Une Analyse Factorielle des Correspondances (AFC) a mis en exergue les relations espèces-organes. Les fréquences de citation, l'indice de fidélité, les prix de vente ont démontré l'importance socio-économique des espèces. Les résultats ont révélé 62 espèces comestibles réparties dans 31 familles et 54 genres. Ces espèces, dominées par les Annonaceae, Apocynaceae, avaient une diversité générique plus marquée chez les Anacardiaceae. Leur exploitation répondait essentiellement à des besoins alimentaires (53\%), énergétique (16\%), technologique $(12 \%)$, médicinal $(12 \%)$. Les AFC ont révélé trois groupes d'utilisation des organes avec une forte corrélation entre fruit et racine d'une part, et feuille, écorce et bois d'autre part. L'étude a également identifié 16 espèces commercialisées. Le fruit était le principal organe consommé et commercialisé. Le plus grand nombre d'organes commercialisés était enregistré chez Elaeis guineensis (4 organes) et Borassus aethiopium (3). Ces produits, souvent collectés et vendus par les femmes et les enfants, provenaient des villages et transitaient dans les marchés par le biais des « bana 
bana ». Leurs prix dépendaient de l'enclavement et de la période de production. Ces résultats ouvrent des perspectives pour une meilleure organisation des prélèvements et de la commercialisation des produits forestiers.

Mots-clés: Espèces comestibles, organes, commercialisation, Zone Kasa, Sénégal

\section{Introduction}

En Afrique, les espèces fruitières sauvages fournissent aux populations locales des produits alimentaires, médicinaux, technologiques de bases pour satisfaire leurs besoins primaires. Leur utilisation est plus accrue en milieu rural où beaucoup de personnes dépendent d'elles pour leurs besoins de subsistance (Bikoué \& Essomba, 2007 ; Malela, 2016). En outre, les produits végétaux non ligneux procurent des revenus substantiels pour bon nombre de ménages (Dieng, 2017). En effet, en plus du bois qui est exploité pour couvrir les besoins énergétiques et de construction en milieu rural (Sambou, 2004 ; Dieng et al., 2016a), le fruit et les feuilles sont bien commercialisés dans les marchés locaux, nationaux, régionaux et internationaux (El-Siddig et al., 2006 ; Mbaye, 2006 ; Aboubakar et al., 2009 ; Bourou et al., 2010, Kouebou et al., 2013 ; Dieng, 2017). La cueillette, la transformation et la vente de ces produits forestiers permettent ainsi aux ruraux d'acquérir des revenus leur permettant d'acheter des denrées de première nécessité et des outils de production. Selon Apema et al. (2010), la valeur commerciale des produits forestiers non ligneux se situait entre 5 et 10 milliards de dollars américain soit 3110 et 6220 milliards Franc CFA dans les années 1990. Aujourd'hui, face à l'intensité de l'exploitation des produits végétaux forestiers liée à la forte croissance démographique des pays africains et aux efforts consentis dans leur transformation, il se pose le problème de leur exploitation durable. Pour éviter la perte de diversité des espèces comestibles et les répercussions négatives sur la nutrition et les revenus des populations, il est important de faire un état des lieux des ressources végétales disponibles afin de pouvoir dégager des stratégies de gestion durable. Cette étude vise à identifier les espèces ligneuses comestibles en zone Kasa (département d'Oussouye, Sénégal) et à étudier leur importance socio-économique. Pour ce faire, plusieurs objectifs spécifiques sont définis. Il s'agit : 1) d'inventorier toutes les espèces comestibles de la zone Kasa et leurs utilisations ; 2) d'identifier les organes commercialisés ; et 3) d'identifier les circuits de commercialisation des divers produits tirés de ces espèces. 


\section{Méthodologie \\ Site d'étude}

Le département d'Oussouye est situé au Sud-Ouest du Sénégal et couvre $891 \mathrm{~km}^{2}$ soit $12,14 \%$ de la superficie de la région de Ziguinchor. Il est limité à l'Est par le marigot de Kamobeul, au Nord par le fleuve Casamance, à l'Ouest par l'Océan Atlantique et le Cap Roxo, au Sud par la Guinée Bissau. Il comprend cinq communes qui totalisent soixante-quinze (75) villages, pour une population de 48331 habitants en 2013 (ANSD, 2015).

\section{Echantillonnage et collecte des données}

L'étude a adopté une démarche qui combine une approche quantitative et qualitative. La population d'étude est constituée par les Diola qui forment l'ethnie majoritaire du département d'Oussouye et parlent plusieurs dialectes. Dans le cas où le dialecte n'est pas maitrisé par l'enquêteur, les services d'un interprète ont été loués afin d'assurer une bonne communication.

Le choix des villages où devaient s'effectuer les enquêtes a été réalisé en tenant compte de la présence de ressources forestières qui permettent les activités de cueille et/ou de leur situation de carrefour commercial des produits forestiers végétaux. Sur la base de ces critères, trente-quatre (34) villages ont été retenus pour la collecte des données. Ces villages sont répartis dans cinq (5) communautés traditionnelles (An Alufay, Esulalu, Ejamat, Dyiwat et Her ou Haer). Chaque communauté se distingue par son dialecte, son organisation et ses pratiques socioculturelles.

Le choix des personnes à enquêter a été basé sur une approche non probabiliste et leur identification a été faite par convenance ou en boule de neige. Pour l'échantillonnage par convenance, le choix a été réalisé avec l'aide du chef de village et a ciblé des individus dont l'activité, l'expérience ou le statut est en rapport avec la thématique. Les entretiens ont été réalisés en 2014 majoritairement auprès des autochtones hommes majeurs, de préférence, qui ont une meilleure connaissance des espèces sauvages.

Les données ont été collectées au cours d'entretiens semi-structurés et des conversations anodines. Les interviews semi-directes (individuels et de groupe) ont été effectués à l'aide d'un guide d'entretien et ont ciblé les personnes ayants pratiqué au moins une fois une activité commerciale en rapport avec les produits forestiers en zone Kasa. Le guide d'entretien comportait les rubriques suivantes : les plantes fruitières comestibles et leurs utilisations, les espèces comestibles commercialisées, les organes commercialisés, les prix et lieux de vente du produit. A chaque fois que de besoin, des échantillons de plantes ont été prélevés pour une identification. Les conversations anodines occasionnelles ont permis à la fois d'évaluer les connaissances et de recueillir de nouvelles données (Martin, 1995). Elles ont surtout été appliquées dans les centres commerciaux car l'usage du guide 
d'entretien risquait de susciter des réticences chez les commerçants. A chaque occasion, les prix des produits commercialisés et les unités de mesure ont été recueillis ; très souvent, des produits ont été achetés afin de déterminer le prix au kilogramme.

Le nombre de personnes interrogées par zone d'étude et par village dépendait de leur disponibilité. Au total, quatre-vingt-deux (82) informateurs ont été interrogés dans les cinq zones traditionnelles (Tableau 1). Le classement des informateurs en classe d'âge et de sexe a permis d'obtenir un ratio homme/femme de $80,77 \%$ contre $19,23 \%$ pour les femmes avec un âge minimum de quinze (15) ans. Ces informateurs ont été interrogés soit individuellement soit en groupe. Le nombre de personne interviewé par groupe variait entre deux (2) et dix (10) ce qui fait un total de 32 groupes et 50 personnes interrogées individuellement. Chaque personne a été invitée à répondre de façon exhaustive aux questions en langue locale.

Tableau 1 : Nombre de villages et proportion des informateurs selon la subdivision administrative et leur communauté traditionnelle

\begin{tabular}{|c|c|c|c|c|c|c|}
\hline \multirow[t]{2}{*}{$\begin{array}{l}\text { Arrondiss } \\
\text { ement }\end{array}$} & \multirow[t]{2}{*}{ Commune } & \multirow[t]{2}{*}{$\begin{array}{l}\text { Nombre de } \\
\text { village }\end{array}$} & \multirow[t]{2}{*}{$\begin{array}{l}\text { Nombre de } \\
\text { village visité }\end{array}$} & \multirow[t]{2}{*}{$\begin{array}{l}\text { Communautés } \\
\text { traditionnelle }\end{array}$} & \multicolumn{2}{|c|}{$\begin{array}{l}\text { Nombre } \\
\text { d'informateurs }\end{array}$} \\
\hline & & & & & $\begin{array}{l}\text { En } \\
\text { groupe }\end{array}$ & $\begin{array}{l}\text { Individ } \\
\text { uels }\end{array}$ \\
\hline & Oussouye & 2 & 1 & \multirow[t]{2}{*}{ An alufay } & \multirow[t]{2}{*}{11} & \multirow[t]{2}{*}{27} \\
\hline \multirow{2}{*}{$\begin{array}{l}\text { Loudia } \\
\text { Ouolof }\end{array}$} & Oukout & 19 & 12 & & & \\
\hline & Mlomp & 19 & 6 & Esulalu & 9 & 3 \\
\hline \multirow[t]{3}{*}{ Kabrousse } & $\begin{array}{l}\text { Santhiaba } \\
\text { Manjacque }\end{array}$ & 15 & 10 & Ejamat & 11 & 7 \\
\hline & \multirow[t]{2}{*}{ Diembéring } & \multirow[t]{2}{*}{20} & \multirow[t]{2}{*}{5} & Dyiwat & 3 & 7 \\
\hline & & & & Her ou Haer & & 4 \\
\hline
\end{tabular}

\section{Traitement des données}

Le dépouillement des données a été effectué avec le logiciel d'analyse statistique Epi Info et les données collectées ont été traitées et représentées graphiquement grâce au tableur Excel.

Une Analyse Factorielle des Correspondances (AFC) a été réalisée avec le logiciel R (R Core Team, 2017) avec le package factoextra (Version: 1.0.5) pour étudier les relations entre les différentes espèces et les organes utilisés.

L'identification des plantes a été faite soit sur place, soit au laboratoire de botanique de l'IFAN et à l'herbier de l'université Cheikh Anta Diop de Dakar. Les différentes flores illustrées du Sénégal de Berhaut (1979 ; 1976 : 1975 a et b ; 1974 ; 1971 et 1967), la flore de Hutchinson et Dalziel (1954), les travaux de Adam (1970), de Hawthorne et Jongkind (2006), de Arbonnier (2002) ont permis d'identifier les noms scientifiques des différentes espèces ainsi que leur nom vernaculaire. L'alphabet diola codifié en référence à la langue locale de la communauté de An Alufay a aussi été utilisé. 
La nomenclature utilisée est celle de Lebrun \& Stork (1991 ; 1992 ; 1995 ; 1997). La base de données du Conservatoire et Jardin Botanique (C.J.B) de la ville de Genève a également été consultée pour la vérification de la taxonomie des espèces.

Les différentes utilisations des espèces comestibles ont été rangées dans six (6) catégories. La catégorie «alimentation » regroupe toutes les utilisations liées à l'alimentation humaine ; celle médicinale fait référence à toutes les utilisations curatives et préventives des plantes. La catégorie « énergie » renvoie surtout au bois d'énergie et dans une moindre mesure au charbon de bois. La catégorie technologique regroupe toutes les utilisations liées à l'artisanat, à la construction, à la conservation et à la transformation de certains produits. Les utilisations agronomiques regroupent les espèces dont certains organes sont utilisés pour améliorer la qualité terres agricoles. Enfin, la catégorie culturelle rassemble les pratiques liées aux rites et aux cultes.

L'importance socio-économique des produits issus des espèces sauvages étudiés a été estimée à partir de l'indice de fidélité (IF) et en tenant compte des revenus engrangés par leur commercialisation. L'IF, qui est le pourcentage d'informateurs ayant cité l'usage d'une espèce (ou organe) donnée dans la commercialisation, a été calculé selon la formule de Begossi (1996) et Trotte et Logan (1986) : IF (\%) = (Ip/Iu) x 100

- Ip est le nombre d'informateurs ayant affirmé l'utilisation d'une espèce (organe) dans une catégorie;

- Iu est le nombre total d'informateurs interrogés.

La valeur de l'IF varie de 0 à 100 . Une valeur nulle $(0)$ indique que l'espèce (ou l'organe) n'est pas utilisée tandis qu'un IF de $100 \%$ révèle que l'espèce (organe) est utilisée par tous les enquêtés.

\section{Résultats}

\section{I.1) Espèces fruitières sauvages comestibles de la zone Kasa}

Les données collectées ont permis d'inventorier 62 espèces végétales fruitières comestibles sauvages réparties dans 31 familles. Les Apocynaceae et les Annonaceae sont les plus représentées avec 6 espèces chacune. Elles sont suivis par les Anacardiaceae (5 espèces) et les Chrysobalanaceae, Arecaceae et les Rubiaceae (4 espèces chacune). Les autres familles sont constituées par les Sapindaceae, les Caesalpiniaceae, les Moraceae, les Celastraceae (3 espèces chacune), les Bombacaceae et les Zingiberaceae (2 espèces). Les dix-neuf (19) familles restantes sont représentées chacune par une (1) espèce (Figure 1). 


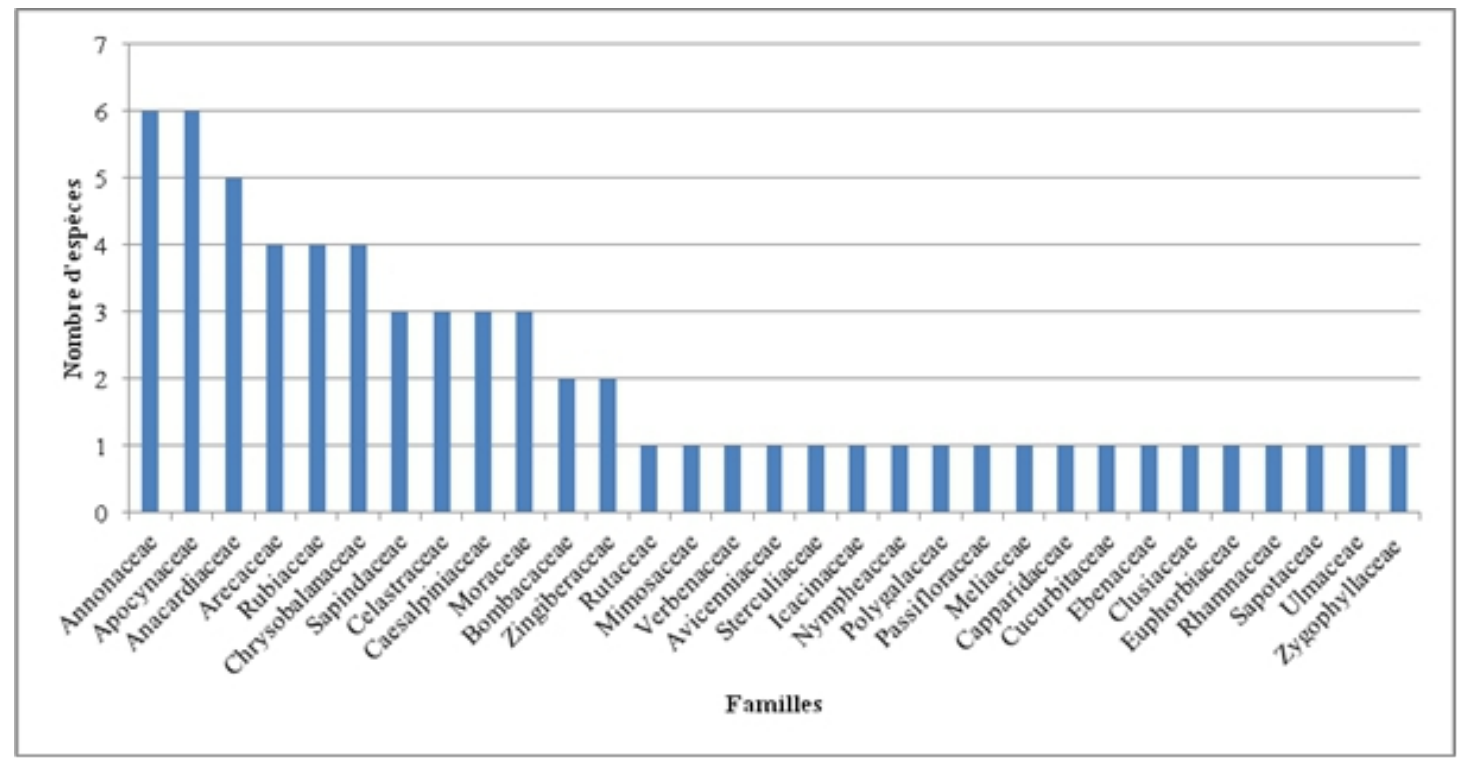

Figure 1 : Diversité des espèces au sein des familles

La diversité générique est également importante avec 54 genres au total. La famille des Anacardiaceae est la plus diversifiée avec 5 genres, suivie de celles des Annonaceae, Arecaceae et Rubiaceae avec 4 genres chacune, des Apocynaceae, Chrysobalanaceae, Sapindaceae, Celastraceae, Caesalpiniaceae avec 3 genres et des Moraceae, Bombacaceae avec 2 genres chacune. Le reste des familles est représenté par un seul genre chacune.

\section{I.2) Différents usages des espèces comestible de la zone Kasa}

L'exploitation des espèces fruitières comestibles porte sur différents organes et varie en fonction de l'espèce. Le plus grand nombre de citations d'usage concerne Elaeis guinenesis, Borassus aethiopum, Mangifera indica, Neocarya macrophylla, Parkia biglobosa et Anacardium occidentale. Parmi les espèces citées, Elaeis guineensis et Mangifera indica sont celles qui renferment les plus de parties exploitées, soit six (6) parties par espèce. Toutes les espèces recensées sont exploitées pour leurs fruits. Les feuilles, le bois et les racines sont aussi exploités chez plus de $50 \%$ des espèces comestibles. Un total de 178 utilisations ont été rapportées par les enquêtés. Ces utilisations entrent dans six (6) catégories d'usage (figure 2). Les espèces sont plus utilisées dans la catégorie alimentaire (53\%), suivie des catégories énergétique (16\%), technologique (12\%) et médicinal (12\%). 


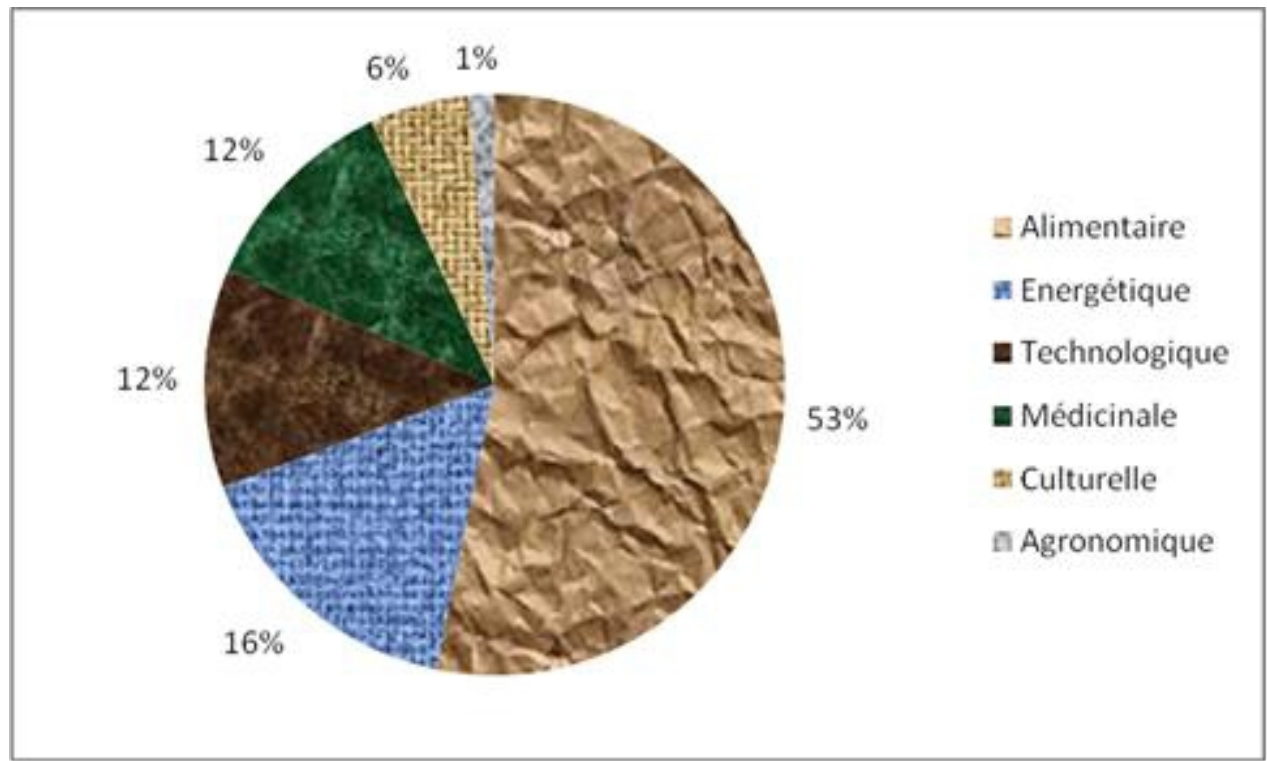

Figure 2. Fréquences relatives des catégories d'usage des espèces comestibles

Dans la catégorie «alimentation », les fruits de toutes les espèces sont concernés. Il s'y ajoute les exsudats et la fleur de Elaeis guineensis, les racines de Nymphaea sp., les feuilles de Adansonia digitata, le gemmule de Borassus aethiopum et l'écorce de Landolphia dulcis.

Dans la catégorie énergétique, quatre organes des 26 espèces sont concernés. La tige est prélevée chez toutes ces espèces. Elle est suivit de la feuille de Elaeis guineensis, de la fleur de Borassus aethiopum et du fruit de Parkia biglobosa. Ces organes sont directement utilisés pour fournir du feu de cuisine aux ménages tandis que la tige est parfois utilisée pour faire du charbon de bois.

Dans la catégorie technologique, 34 espèces sont inventoriées et leur utilisation concerne six organes. La tige de Mangifera indica et Ceiba pentendra est utilisée pour la confection des pirogues et des abreuvoirs des porcs ; les feuilles de Elaeis guineensis pour la construction de clôtures ; les fruits immatures de Neocarya macrophylla pour la teinture des filets; l'exsudat de Landolphia heudelotii comme colle, l'écorce de Uvaria chamae pour la décoration de vanneries; la racine de Landolphia dulcis accélère la fermentation du vin de palme.

Dans la catégorie médicinale, 52 espèces sont concernées pour tous les organes exploités. Les espèces les plus utilisées sont: Mangifera indica, Elaeis guineensis, Xylopia aethiopica, Parkia biglobosa, Sarcocephalus latifolius. La tige de Landolphia dulcis est utilisée dans hygiène buccodentaire, le décocté des feuilles de Mangifera indica pour le traitement du paludisme, consommation des fruits de Parkia biglobosa pour soigner la 
diarrhée, le macéré des racines de Sarcocephalus latifoliu pour traiter les douleurs abdominales, l'exsudat de Landolphia heudelotii pour traiter les crachats du cobra africain tombés dans les yeux, le macéré de la fleur de Elaeis guineensis pour traiter le fibrome, le macéré de l'écorce de Parkia biglobosa dans le traitement de l'anémie.

Dans la catégorie culturelle, 9 espèces sont recensés. Les feuilles de Elaeis guineensis et de Phoenix reclinata sont utilisées dans la danse et comme masque des initiés, la tige de Voacanga africana est utilisée pour la décoration des têtes des lutteurs, l'exsudat ou vin de palme de Elaeis guineensis est utilisé pour la vénération des fétiches (bəkin) et pour la quasi-totalité des pratiques traditionnelles (dot, libations, funérailles, etc.). L'écorce de Adansonia digitata accompagne la représentation symbolique du fétiche des morts « $\mathrm{ku}$ huluy », le tronc de Ceiba pentandra sert à la confection de cercueil.

Dans le domaine agronomique, 10 espèces sont retrouvées. Les feuilles de Mangifera indica, et de Ceiba pentandra, et les fruits de Parkia biglobosa sont souvent utilisés comme fertilisants des sols cultivés.

Le tableau 2 présente les inerties de chacun des axes factoriels obtenus après transformation des variables d'origine (espèce et organe). Le pourcentage d'inertie des trois (3) premières dimensions est supérieur au seuil moyen 1/6 $=17 \%$. Les trois premières dimensions cumulent plus de $70 \%$ de l'inertie totale portée par les variables d'origine. Ce seuil d'inertie parait suffisant pour tirer des conclusions importantes.

Tableau 2 : Répartition des inerties suivant les dimensions (dim)

\begin{tabular}{|c|c|c|c|c|c|c|}
\hline & $\operatorname{dim} 1$ & $\operatorname{dim} 2$ & $\operatorname{dim} 3$ & $\operatorname{dim} 4$ & $\operatorname{dim} 5$ & $\operatorname{dim} 6$ \\
\hline Inerties & 0,26 & 0,21 & 0,16 & 0,12 & 0,09 & 0,03 \\
\hline Pourcentage d'inerties & 30,05 & 24,06 & 18,15 & 14,18 & 10,58 & 2,98 \\
\hline $\begin{array}{l}\text { Pourcentage } \\
\text { d'inerties }\end{array}$ & 30,05 & 54,11 & 72,26 & 86,44 & 97,02 & 100 \\
\hline
\end{tabular}

Les deux (2) premières dimensions qui expriment $54,1 \%$ de l'inertie totale des variables d'origines permettent de structurer les résultats en trois groupes (figure 3). La dimension 1 horizontale qui exprime $30 \%$ de l'inertie totale permet d'isoler le groupe 1 des groupes 2 et 3 . Le groupe 1 caractérise deux espèces dont les organes utilisés sont la fleur et l'exsudat. Le groupe 2 regroupe les espèces dont le bois, l'écorce et la feuille sont les plus utilisés tandis que le groupe 3 est constitué d'espèces dont les fruits et les racines sont les plus exploités.

Le groupe 2 regroupe les espèces à organes utilisés opposées au groupe 3. Ces deux groupes sont présentés par la dimension 2, verticale qui exprime $24,1 \%$ de l'inertie totale. La dimension 3 qui exprime 18,2\% de l'inertie totale, permet de structurer chacun des groupes 2 et 3 en deux sous-groupes (figure 4). Le groupe 2 est subdivisé en sous-groupe d'espèces à usage de bois et d'espèces à usage d'écorce. 
Le groupe 3 comprend le sous-groupe d'espèces à usage des racines et le sous-groupe d'espèces à usage de fruits. Cela montre que l'utilisation de la racine est toujours corrélée avec celle du fruit, d'une part et l'utilisation de l'écorce et du bois à celle de la feuille, d'autre part.

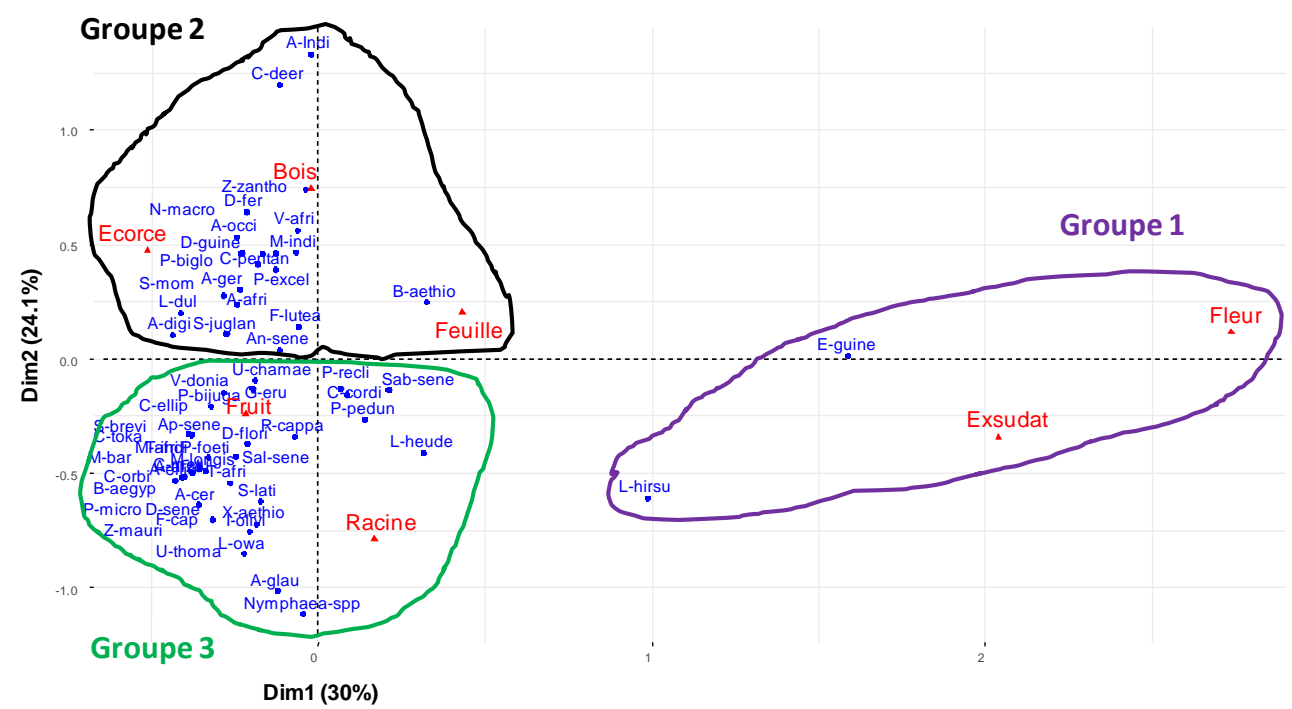

Figure 3 : Analyse Factorielle des Correspondances (AFC) croisant la variable Espèce (62 espèces) et la variable Organe (7 organes exploités) dans le plan factoriel Dim1xDim2.

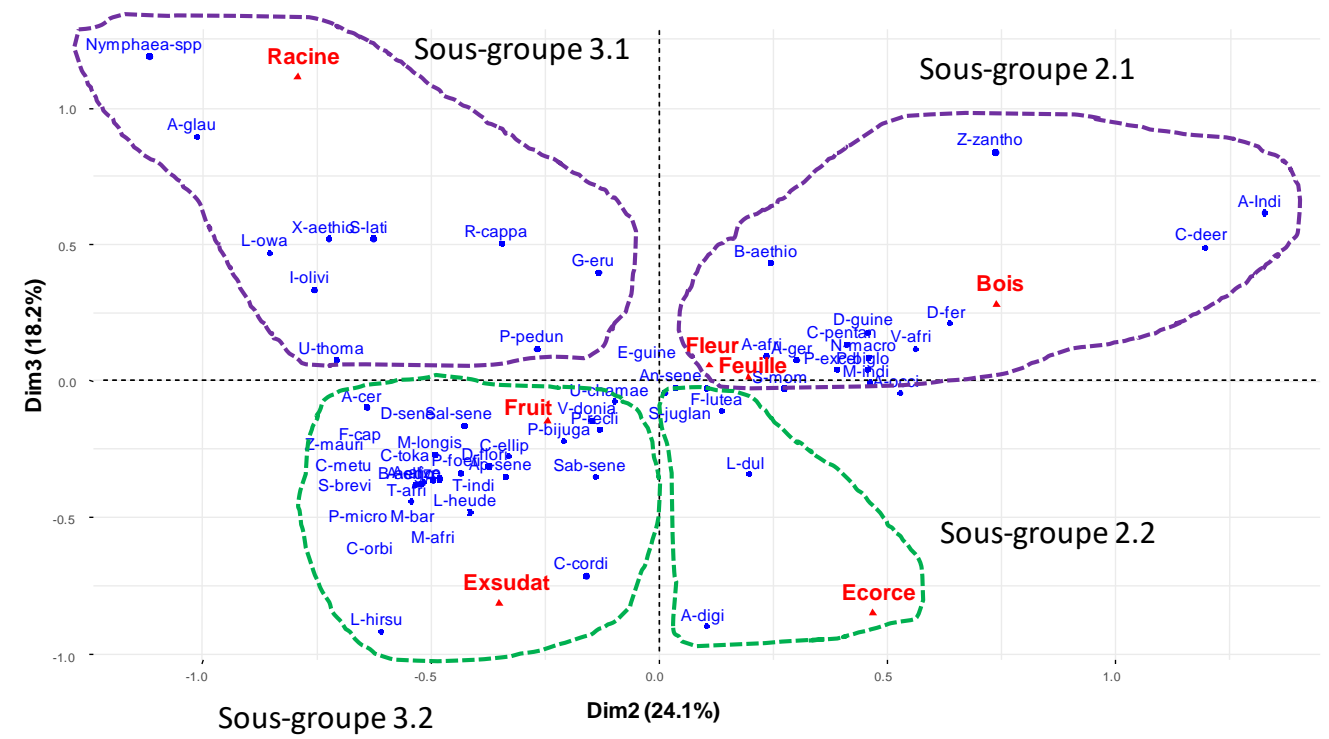

Figure 4 : Analyse Factorielle des Correspondances (AFC) des 62 espèces x 7 organes exploités dans le plan des axes Dim2xDim3. 


\section{I.3) Espèces fruitières sauvages commercialisées de la zone Kasa}

Les résultats (tableau 3) montrent que 16 des 62 espèces comestibles identifiées (soit 26\%) sont commercialisées. Les produits vendus sont principalement les fruits (73\% des citations), les feuilles (14\%) et les exsudats $(12 \%)$ et secondairement le bois (2\%). Au total quatre (4) organes sur les sept (7) sont collectés et vendus par la population. Les espèces qui présentent le plus grand nombre d'organes commercialisés sont : Elaeis guineensis $(89 \%$ des citations) et Borassus aethiopium (7\% des citations) qui totalisent respectivement 4 et 3 organes : il s'agit des fruits, des feuilles, des exsudats et du bois (ou gemmule). Pour les quatorze (14) autres espèces, seul le fruit est commercialisé. A l'exception des palmiers, le bois des ligneux est parfois transformé en charbon qui est vendu dans le secteur.

Tableau 3 : liste des organes ou produits commercialisés et leurs indices de fidélité

\begin{tabular}{|c|c|c|c|c|c|}
\hline \multirow{2}{*}{ Espèces } & \multirow{2}{*}{ Nom local } & \multicolumn{4}{|c|}{ indices de fidélité } \\
\hline & & $\mathbf{F r}$ & $\mathbf{F e}$ & Bo & $\mathbf{E x}$ \\
\hline Mangifera indica L. "Perse" & $\begin{array}{l}\text { bu mangali bu jóoluay, bu } \\
\text { mangu bu jóoluay }\end{array}$ & $9 \%$ & & & \\
\hline Ceiba pentandra (L.) Gaertn & bu sana & $9 \%$ & & & \\
\hline Elaeis guineensis Jacq. & ka bekel, ka hiit & $76 \%$ & $73 \%$ & $2 \%$ & $89 \%$ \\
\hline Adansonia digitata $\mathrm{L}$. & bu koya, bu baak & $51 \%$ & & & \\
\hline Parkia biglobosa (Jaq.) Benth. & bu nalay, bu níók & $48 \%$ & & & \\
\hline $\begin{array}{l}\text { Saba senegalensis (A. DC.) } \\
\text { Pichon }\end{array}$ & bu híndik, bundok & $66 \%$ & & & \\
\hline Landolphia heudelotii A. DC. & bu hemb & $43 \%$ & & & \\
\hline $\begin{array}{l}\text { Neocarya macrophylla (Sabine) } \\
\text { Prance }\end{array}$ & bu bita, bu yafay, beel & $20 \%$ & & & \\
\hline Parinari excelsa Sabine & bu wel, bu fujay, e liik & $20 \%$ & & & \\
\hline Dialium guineense Willd. & bu fulan, bu foyitay & $20 \%$ & & & \\
\hline Anacardium occidentale L. & bu talakasa, bu bisa & $74 \%$ & & & \\
\hline Borassus aethiopum (L.) Mart. & ka lahaay & $6 \%$ & $5 \%$ & $7 \%$ & \\
\hline $\begin{array}{l}\text { Aphania senegalensis (Juss. Ex } \\
\text { Poir.) Radlk. }\end{array}$ & bu ul & $4 \%$ & & & \\
\hline Vitex doniana $\mathrm{Sw}$. & bu jink, bu kuf & $18 \%$ & & & \\
\hline $\begin{array}{l}\text { Xylopia aethiopica (Dunal) A. } \\
\text { Rich. }\end{array}$ & bu lewbafinoe & $5 \%$ & & & \\
\hline Tamarindus indica $\mathrm{L}$. & bu dahar & $2 \%$ & & & \\
\hline
\end{tabular}

Dans la zone Kasa, la gestion et la collecte des produits forestiers se font de plusieurs manières. Dans les terrains privés, la gestion et la collecte des espèces à forte valeur économique comme Adansonia digitata, Anacardium occidentale, sont rigoureuses et efficientes. Toutefois, le statut privé de ces espèces pose problème et occasionne souvent des conflits au sein des populations. Dans les domaines communautaires, la gestion et la collecte sont libres. La pression anthropique sur les espèces telles que Mangifera indica, Parinari excelsa, Aphania senegalensis, Vitex doniana et Xylopia 
aethiopica est moins intense du fait de leur faible valeur marchande. La collecte de leurs organes n'est pas réglementée.

\section{I-3) Les circuits de commercialisation et les acteurs}

La commercialisation de ces produits forestiers s'effectue à quatre (4) différentes échelles. Elle peut se faire dans les villages de collecte, dans la capitale départementale (Oussouye), dans la station balnéaire du Cap Skirring (figure 5) et enfin dans la capitale régionale (Ziguinchor) ou nationale (Dakar).

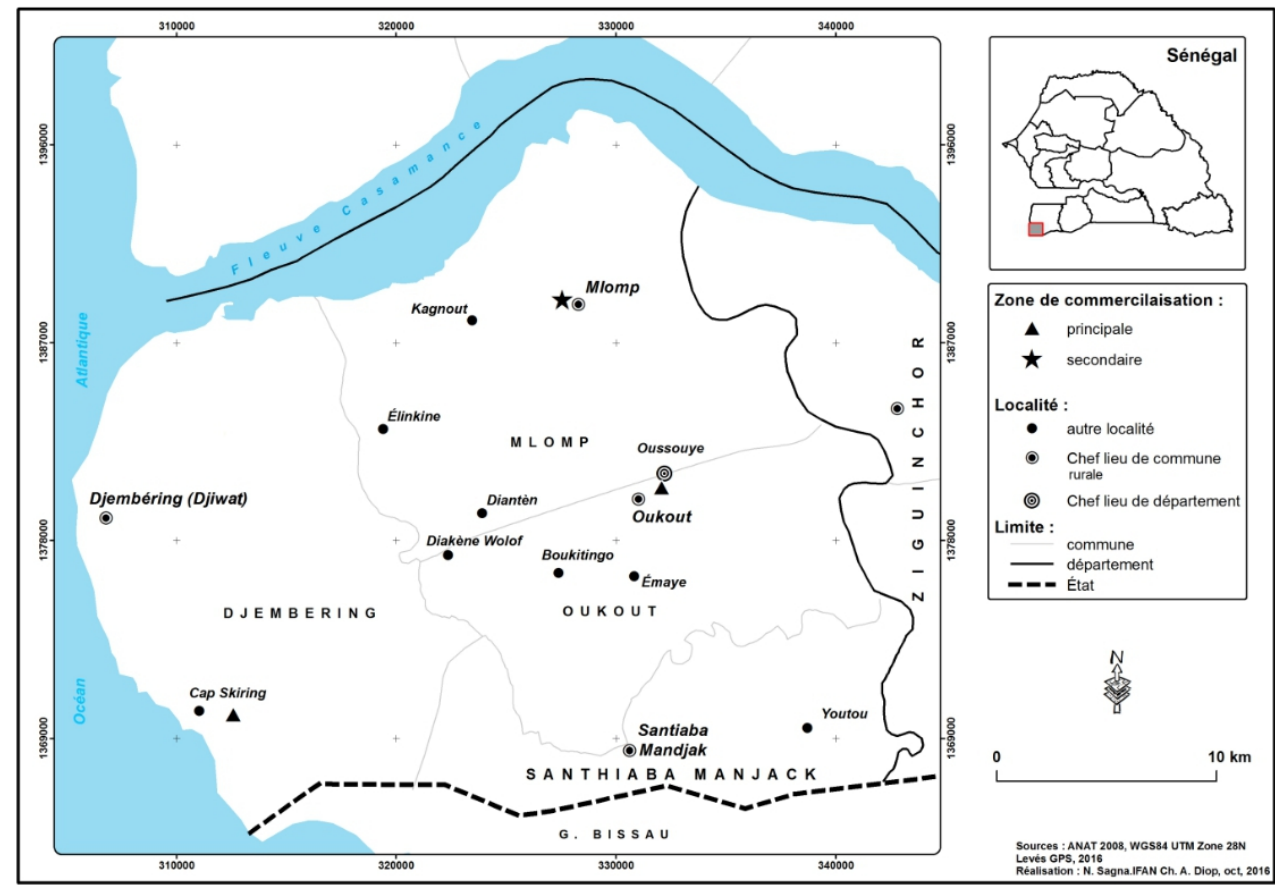

Figure 5 : Les principales zones de commercialisation des produits forestiers du département d'Oussouye.

La commercialisation à l'échelle départementale est plus répandue. En effet, les produits sont d'abord acheminés vers le village le plus proche auprès d'un notable commerçant appelé tuteur qui se chargera de la vente. Les produits forestiers sont d'abord destinés au marché départemental d'Oussouye et au centre touristique du Cap Skirring. Les capitales régionales comme Ziguinchor et Dakar, ne reçoivent que la quantité excédentaire ou les produits moins prisés dans la zone. Les collecteurs vendent le produit directement aux consommateurs ou à des groupes intermédiaires, commerçants et grossistes auprès desquels les détaillants viennent se ravitailler (figure 6). 


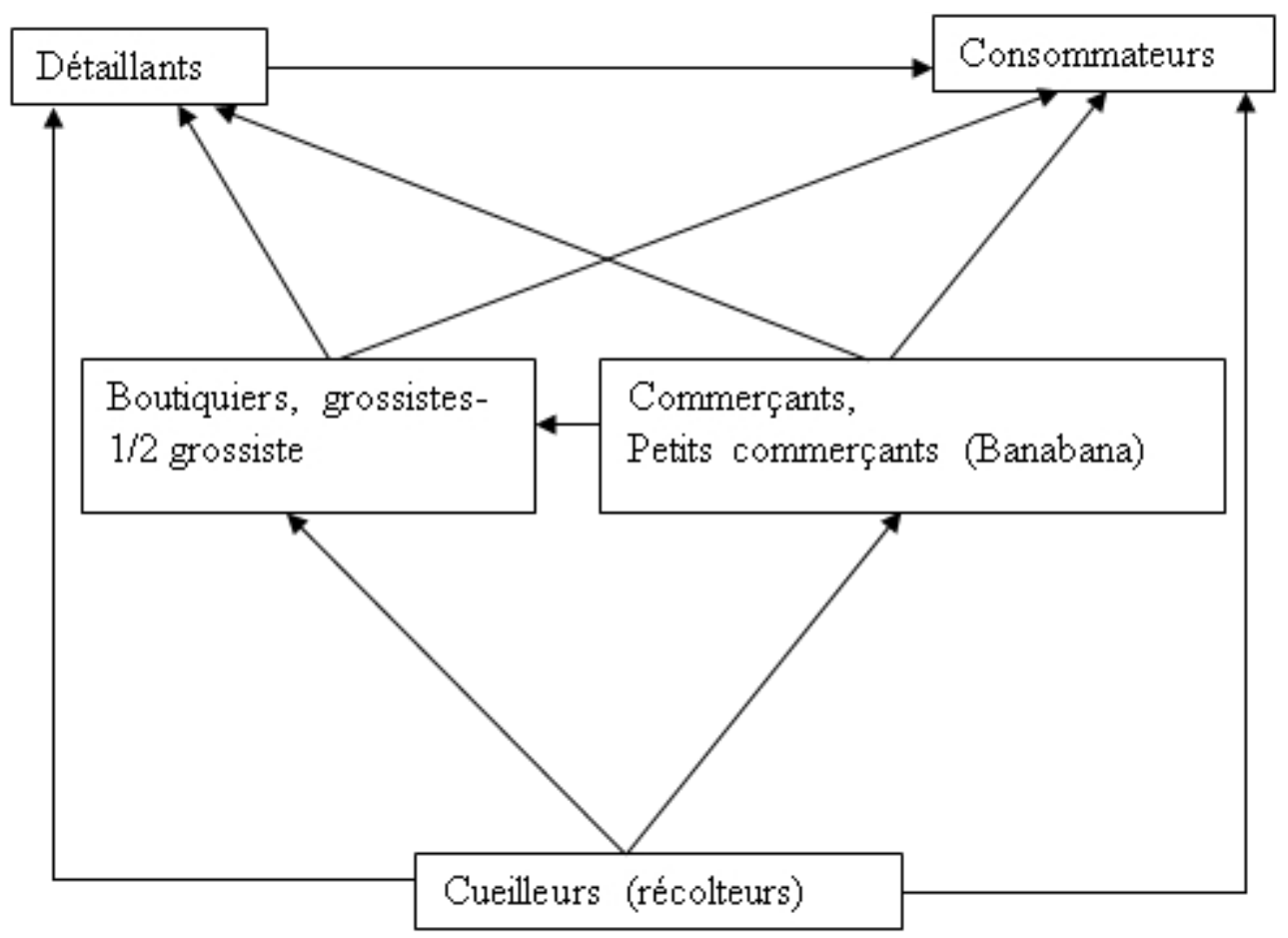

Figure 6 : Les acteurs et les circuits de commercialisation des fruits comestibles sauvages

Les acteurs de la vente des produits forestiers sont :

$>$ Les cueilleurs, qui sont hommes et femmes de tout âge, récoltent les produits des végétaux sauvages dans des zones biens circonscrites et les revendent aux commerçants. Ils peuvent effectuer des opérations simples chez eux (séchage, nettoyage, transformation primaire). Ils disposent souvent d'un contrat verbal directement avec les commerçants pour leur livrer des produits de qualité.

$>$ Les petits commerçants appelés «bana bana» sont composés majoritairement des femmes. Ils vont dans les villages à la recherche des produits et les acheminent vers les centres commerciaux de la zone Kasa, de Ziguinchor et des autres régions. Ces commerçants nouent des relations clientélistes avec les villageois en faisant du porte à porte et achètent le produit dans des marchés villageois. Ils achètent les produits aux cueilleurs, les stockent et les revendent aux boutiquiers. Les produits sont souvent pris à crédit par les commerçants dont les moyens financiers sont faibles et le payement se fera aux prochains embarquements. Les «bana bana » et les cueilleurs mutualisent souvent leurs efforts pour assurer le transport des produits forestiers vers les centres commerciaux où ils doivent s'acquitter du payement des taxes communales. L'acheminement de ces produits vers les lieux de vente est soumis au respect des règles du code forestier. 
$>$ Les boutiquiers ou grossistes sont essentiellement des hommes. Ils achètent les produits en grande quantité puis les revendent à des commerçants détaillants ou les exportent vers les autres régions. On peut rencontrer d'anciens « bana bana » reconvertis en commerçants sédentaires et des relations clientélistes avec les cueilleurs ou même les « bana bana ».

$>$ Les détaillants sont essentiellement constitués des femmes. Ils profitent de la disponibilité des produits qu'ils soient agricoles ou forestiers. Ils se procurent des produits auprès des collecteurs, des «bana bana »et des grossistes. Ils peuvent les transformer et les stocker. Ces produits sont ensuite vendus aux consommateurs dans les centres commerciaux ou lieux aménagés.

Le tableau 4 montre que la commercialisation de l'espèce peut concerner un organe en entier (bois, feuille, exsudat) ou une partie du fruit (la graine, la pulpe et l'amande). Le nombre de parties du fruit vendues varient selon les espèces :

- trois chez Elaeis guineensis (fruit entier, pulpe, graine), Anacardium occidentale (fruit entier, pulpe, amande), Parkia biglobosa (fruit entier, pulpe, graine) ;

- deux chez Landolphia heudelotii (fruit entier, jus de condiment de la pulpe), Mangifera indica (fruit entier, boisson alcoolisée) ;

- une seule partie, le fruit chez les neuf espèces, l'amande chez Neocarya macrophylla et l'épicarpe du fruit (utilisé condiment) chez Ceiba pentandra.

La collecte des produits commercialisés est assurée par 4 groupes sociaux d'âge et de sexe différents. Il s'agit des jeunes hommes, des jeunes femmes, des enfants et des vieillards (tableau 5). Les résultats montrent que la commercialisation des produits forestiers est assurée aussi bien par les hommes que par les femmes. Ces acteurs appartiennent à :

- deux tranches d'âge (adulte et enfant) pour les espèces comme Saba senegalensis, Landolphia heudelotii, Dialium guineense et Anacardium occidentale ;

- une seule tranche d'âge (adulte ou enfant) pour le reste des espèces.

Les prix de vente varient suivant le rapport de l'offre et de la demande. Ils dépendent aussi de la nature (transformée ou brute) des articles proposés, de l'enclavement de la zone de cueillette et des frais de transport. Ainsi, lorsqu'on s'éloigne des lieux de cueillette (village), les prix augmentent. Par contre, le nombre de produits vendus diminue avec l'éloignement des lieux de collecte et certains sont même absents dans les marchés communaux, 
touristiques, régionaux ou de la capitale (tableau 4). Le sac (50 kg de riz) et les pots (d'une contenance de $100 \mathrm{~g}$ de café) sont les principales unités de mesures utilisées pour la commercialisation des produits forestiers. Le kilogramme et la bouteille d'un litre sont aussi utilisés.

Tableau 4 : Prix des produits forestiers en fonction du site de commercialisation

\begin{tabular}{|c|c|c|c|c|c|}
\hline \multirow[t]{2}{*}{ Espèces } & \multirow[t]{2}{*}{ collecteur } & \multirow[t]{2}{*}{ Organes } & \multirow{2}{*}{$\begin{array}{l}\text { Produit } \\
\text { commercialisé }\end{array}$} & \multicolumn{2}{|l|}{ Prix de vente } \\
\hline & & & & Au village & $\begin{array}{l}\text { Oussouye ou } \\
\text { Cap Skirring }\end{array}$ \\
\hline \multirow{2}{*}{ Mangifera indica } & \multirow[t]{2}{*}{$\mathrm{G}$} & \multirow{2}{*}{$\mathrm{Fr}$} & $\mathrm{Fr}$ & 25 à $50 \mathrm{~F} / \mathrm{tas}$ & \\
\hline & & & B.al. & $100 \mathrm{~F}$ à $300 \mathrm{~F} / \mathrm{L}$ & \\
\hline Ceiba pentandra & $\mathrm{V}$ & $\mathrm{Fr}$ & Condiment (sel) & 750 à $1000 \mathrm{~F} / \mathrm{bou}$ & \\
\hline \multirow[t]{6}{*}{ Elaeis guineensis } & \multirow[t]{6}{*}{$\mathrm{H}$} & \multirow[t]{3}{*}{$\mathrm{Fr}$} & $\mathrm{Fr}$ & 25 à $100 \mathrm{~F} /$ pot & 75 à $100 \mathrm{~F} /$ pot \\
\hline & & & Huile de palme & 1000 à $1500 \mathrm{~F} / \mathrm{L}$ & $1500 \mathrm{~F} / \mathrm{L}$ \\
\hline & & & Graines & 500 à $1000 \mathrm{~F} / \mathrm{sac}$ & \\
\hline & & $\mathrm{Fe}$ & $\mathrm{Fe}$ & 25 à $100 \mathrm{~F} / \mathrm{tas}$ & $100 \mathrm{~F} / \mathrm{tas}$ \\
\hline & & $\mathrm{Ex}$ & B.al. & 200 à $400 \mathrm{~F} / \mathrm{L}$ & $300 \mathrm{~F} / \mathrm{L}$ \\
\hline & & Bo & Bo & $1000 \mathrm{~F} / \mathrm{stipe}$ & \\
\hline Adansonia digitata & $\mathrm{G}$ & $\mathrm{Fr}$ & $\mathrm{Fr}$ & $\begin{array}{l}4000 \text { à } 7500 \mathrm{~F} / \mathrm{sac} \\
\text { ou } 50 \text { à } 100 \mathrm{~F} / \text { pot }\end{array}$ & $\begin{array}{l}6000-7500 \\
\text { F/sac ou } 75 \text { à } \\
100 \mathrm{~F} / \text { pot }\end{array}$ \\
\hline \multirow[t]{3}{*}{ Parkia biglobosa } & \multirow[t]{3}{*}{$\mathrm{Fe}$} & \multirow[t]{3}{*}{ Fr } & Graines & $\begin{array}{l}50 \text { à100 F/pot ou } \\
75 \text { à } 250 \mathrm{~F} / \mathrm{kg}\end{array}$ & \\
\hline & & & $\begin{array}{l}\text { graines } \\
\text { fermentées }\end{array}$ & $25 \mathrm{~F} / \mathrm{tas}$ & $25 \mathrm{~F} / \mathrm{tas}$ \\
\hline & & & pulpe en poudre & & $50 \mathrm{~F} /$ pot \\
\hline Saba senegalensis & $\mathrm{Fe}, \mathrm{G}, \mathrm{F}$ & $\mathrm{Fr}$ & $\mathrm{Fr}$ & $\begin{array}{l}2000-6000 \mathrm{~F} / \mathrm{ba} \text { ou } \\
100-500 \mathrm{~F} / \mathrm{tas}\end{array}$ & $\begin{array}{lll}8000 & \text { à } & 10000 \\
\text { F/sac } & & \\
\end{array}$ \\
\hline \multirow{2}{*}{$\begin{array}{l}\text { Landolphia } \\
\text { heudelotii }\end{array}$} & \multirow[t]{2}{*}{$\mathrm{Fe}, \mathrm{G}, \mathrm{F}$} & \multirow[t]{2}{*}{$\mathrm{Fr}$} & Fr & 1500 à $3500 \mathrm{~F} / \mathrm{ba}$ & \\
\hline & & & Jus fermenté & 250 à $500 \mathrm{~F} / \mathrm{L}$ & \\
\hline $\begin{array}{l}\text { Neocarya } \\
\text { macrophylla }\end{array}$ & $\mathrm{V}$ & $\mathrm{Fr}$ & $\mathrm{Am}$ & 200 à $250 \mathrm{~F} / \mathrm{pot}$ & 200 à $500 \mathrm{~F} /$ pot \\
\hline Parinari excelsa & $\mathrm{G}, \mathrm{F}$ & $\mathrm{Fr}$ & $\mathrm{Fr}$ & $50 \mathrm{~F} / \mathrm{pot}$ & $50 \mathrm{~F} / \mathrm{pot}$ \\
\hline Dialium guineense & $\mathrm{Fe}, \mathrm{G}, \mathrm{F}$ & $\mathrm{Fr}$ & Fr & $\begin{array}{l}2000 \text { à } 7500 \mathrm{~F} / \mathrm{sac} \\
\text { ou } 50 \mathrm{~F} / \mathrm{pot}\end{array}$ & $\begin{array}{l}25 \text { à } 100 \mathrm{~F} / \mathrm{pot} \\
\text { ou } 7500 \mathrm{~F} / \mathrm{sac}\end{array}$ \\
\hline \multirow{3}{*}{$\begin{array}{l}\text { Anacardium } \\
\text { occidentale }\end{array}$} & \multirow[t]{3}{*}{$\mathrm{Fe}, \mathrm{G}, \mathrm{F}$} & \multirow[t]{3}{*}{$\mathrm{Fr}$} & $\mathrm{Fr}$ & 100 à $500 \mathrm{~F} / \mathrm{kg}$ & \\
\hline & & & $\mathrm{Am}$ & $\begin{array}{l}100 \mathrm{~F} / 25 \mathrm{~g} \text { ou } 500 \\
\mathrm{~F} / 75 \mathrm{~g}\end{array}$ & $\begin{array}{lll}200 & F / 25 \mathrm{~g} & \text { ou } \\
1000 & F / 75 \mathrm{~g} & \\
\end{array}$ \\
\hline & & & Jus alcoolisé & $100 \mathrm{~F}$ à $200 \mathrm{~F} / \mathrm{L}$ & \\
\hline \multirow[t]{4}{*}{ Borassus aethiopum } & \multirow[t]{4}{*}{$\mathrm{G}$} & Fr & $\mathrm{Fr}$ & $\begin{array}{ll}500 \text { à } & 1000 \\
\text { F/régime } & \\
\end{array}$ & \\
\hline & & $\mathrm{Fe}$ & $\mathrm{Fe}$ & $\begin{array}{l}100 \text { à } 200 \mathrm{~F} / \text { jeune } \\
\text { feuille }\end{array}$ & \\
\hline & & \multirow[t]{2}{*}{ Bo } & Bo & $2.500 \mathrm{~F} / \mathrm{stipe}$ & $\begin{array}{l}3000 \text { à } 4000 \mathrm{~F} / \\
\text { stipe }\end{array}$ \\
\hline & & & Gemmule & 25 F/unité & \\
\hline Aphania senegalensis & $\mathrm{G}, \mathrm{F}$ & $\mathrm{Fr}$ & $\mathrm{Fr}$ & $50 \mathrm{~F} /$ pot & $50 \mathrm{~F} /$ pot \\
\hline Vitex doniana & $\mathrm{G}, \mathrm{F}$ & $\mathrm{Fr}$ & $\mathrm{Fr}$ & $50 \mathrm{~F} / \mathrm{pot}$ & \\
\hline
\end{tabular}




\begin{tabular}{|l|l|l|l|l|l|}
\hline Xylopia aethiopica & $\mathrm{G}$ & $\mathrm{Fr}$ & $\mathrm{Fr}$ & $\begin{array}{l}250 \text { à 300 F/kg ou } \\
100 \mathrm{~F} / \mathrm{pot} \text { ou } 4000 \\
\text { F/sac }\end{array}$ & $800 \mathrm{~F} / \mathrm{kg}$ \\
\hline Tamarindus indica & & $\mathrm{Fr}$ & $\mathrm{Fr}$ & $500 \mathrm{~F} / \mathrm{kg}$ & \\
\hline
\end{tabular}

$\mathbf{F r}=$ fruit $; \mathbf{F e}=$ feuille $; \mathbf{B o}=$ bois $; \mathbf{E x}=$ exsudat $;$ B.al $=$ boisson alcoolisée $;$ Pot $=$ contenance de $100 \mathrm{~g}$ de café $; \mathbf{5 0 k g}=$ sac de $50 \mathrm{~kg}$ de riz $;$ Tas $=$ quantité variable de fruit $; \mathbf{B a}$ = bassine de 30 litres $; \mathbf{B o u}=$ bouteille de 1 litre $; \mathbf{A m}=$ amande $; \mathbf{F e}=$ Femme, $\mathbf{G}=$ garçon ; $\mathbf{F}=$ fille $; \mathbf{V}=$ vieillard.

L'huile de palme de Elaeis guineensis ainsi que la pulpe jaune du fruit de Parkia biglobosa et ses graines bouillies puis fermentées «netetu» sont très appréciées et sont bien commercialisées localement et dans les grandes villes (Ziguinchor et Dakar). Le jus tiré de Mangifera indica après cuisson à l'eau et celui de Anacardium occidentale après pression sont fermentés et donnent une boisson alcoolique très prisée. De l'épicarpe de Ceiba pentandra, on extrait des condiments pour assaisonner le tabac très commun dans le secteur et alimentent le marché local.

\section{Discussion}

\section{Diversité des espèces fruitières consommées et commercialisées}

Les résultats de cette étude ont permis de constater la grande diversité spécifique et générique des espèces fruitières comestibles en zone Kasa. Les 62 espèces identifiées pour cette étude représentent $17,7 \%$ des espèces comestibles de l'Afrique occidentale (Baumer, 1995). Des études antérieures ont signalé 20 espèces commune pour les régions Nord et Est de la Côte d'Ivoire et du Togo (Ouattara et al. 2016; Atato et al. 2010). Le nombre d'espèces comestibles en zone Kasa est supérieur à celui de la Forêt Classée de Patako (Sénégal) où moins vingt-trois (23) espèces comestibles ont été identifiées (Diop, 2011). La diversité spécifique et générique en zone Kasa est également plus importante que celle trouvée dans la réserve forestière de Lama au Bénin où dix (10) espèces sauvages à fruits comestibles ont été dénombrées (Agbahoungba et al., 2016) et à celle du village de Yasikia en RDC où 50 plantes alimentaires sauvages ont été identifiées (Mbula Itumba, 2014). Cette richesse floristique notée peut s'expliquer par la position géographique sub-guinéenne de la zone d'étude. Les résultats montrent une dominance des Apocynaceae et des Annonaceae, et confirment ceux obtenus par Mbula Itumba (2014) en ce qui concerne les Appocynaceae et Ouattara et al. (2016) pour les Annonaceae.

Nos résultats ont également permis d'identifier 16 espèces commercialisées regroupées dans 10 familles. Ce qui montre que dans cette zone, la proportion d'espèces fruitières commercialisées se révèle donc faible comparées aux 25 espèces fruitières sauvages dont les parties sont commercialisées dans les marchés de la ville de Douala (Dibong et al., 2011) 
et aux 29 espèces végétales vendues dans les marchés de Yaoundé (Betti, 2016). Les facteurs déterminants pour une exploitation de ces espèces est leur accessibilité et leur période de disponibilité. La faible diversité des espèces fruitières dans les marchés du département d'Oussouye est liée à une méconnaissance de ces plantes par la population acheteur ou à un changement de comportement alimentaire souvent tourné vers les produits importés.

\section{Utilisations des espèces comestibles}

L'étude a montré que la majorité des espèces répertoriées sont exploitées pour l'alimentation humaine. Près de $2 / 3$ des espèces enregistrent des indices de fidélités supérieurs à $50 \%$ pour leurs fruits. Les fruits cueillis de ces espèces sauvages constituent une part importante dans l'alimentation des Diola du Kasa et contribuent fortement à la sécurité alimentaire pour différentes couches des populations et à la création de richesse. Ils contiennent des glucides, des lipides, des protéines et sont riches en vitamines et en éléments minéraux (Sahoré et al., 2012). Le rôle important des fruitiers locaux dans l'alimentation humaine et la création de richesse au Sénégal est démontré dans de nombreux travaux tels que ceux de Diop (2011), Dieng (2017) et Ndiaye et al. (2017). Par contre, les feuilles des ligneux sauvages sont peu utilisées dans l'alimentation locale en pays Kasa. Les feuilles de Adansonia digitata sont utilisées lors de la préparation des mets comme le couscous sénégalais qui est étranger à la localité et rarement celles de Dialium guineense comme condiment dans la cuisson de la viande. Ces feuilles de Adansonia digitata sont très riches en calcium et en fer et constituent une source appréciable de potassium et de magnésium (Boukari et al., 2001). Contrairement à la zone Kasa où les feuilles de Adansonia digitata sont peu utilisées, dans la zone centre-ouest du Sénégal, elles sont très prisées pour la préparation du couscous sénégalais, un des aliments de base (Ndiaye et al., 2017).

Les autres formes d'usages des espèces comestibles identifiées dans la présente étude concernent la médecine traditionnelle, les pratiques culturelles, l'énergie, etc. Les espèces ligneuses à usage alimentaire jouent un rôle important dans la médecine traditionnelle en Afrique. C'est le cas le notamment de Detarium microcarpum (Kouyaté et al., 2009; Dieng 2016b), de Adansonia digitata (Agbankpé et al., 1014), Cordyla pinnata (Dieng, 2016a) ; Guiera senegalensis (Ndiaye et al., 2017) au Sénégal au Mali et au Bénin. Le bois fait également partie des organes les plus exploités par les populations comme cela a été souligné par Mangambu et al. (2015). En pays Kasa, cinq (5) espèces se singularisent par l'utilisation de leur bois, il s'agit d'une part, de Elaeis guineensis et Borassus aethiopum dont le stipe est très prisé dans la construction des charpentes des habitations comme moyen technologique et d'autre part, de Mangifera indica, Neocarya macrophylla et 
Parinari excelsa dont le bois est plus utilisé comme source d'énergie. Ailleurs, il arrive que le bois d'une seule espèce remplisse à lui seul toutes les fonctions citées ci-dessus. C'est le cas, dans la zone centre-ouest du Sénégal, de Cordyla pinnata dont le bois est très prisé aussi bien pour les charpentes, les clôtures que pour l'énergie (Dieng, 2016a). Les autres organes des espèces comestibles, à savoir l'écorce, la racine, l'exsudat et la fleur sont aussi exploités.

\section{Les acteurs de la commercialisation}

La cueillette des divers organes commercialisés est essentiellement assurée par les femmes et les enfants confirmant les travaux de Alira (2004) sur la commercialisation des fruits sauvages au Burkina Faso. Cependant, lorsque la cueillette requiert beaucoup d'efforts physiques ou lorsqu'elle se fait à grande échelle, ce sont les hommes qui dominent (Dieng, 2017). Les acteurs de la commercialisation ne sont organisés en général, ni en groupe, ni en association. Toutefois la commercialisation des fruits de Landolphia heudelotii, Saba senegalensis, Parkia biglobosa et Anacardium occidentale, apportent de plus en plus de plus-values et occasionne la formation de groupements masculins organisés. Ainsi, la commercialisation fait intervenir un ensemble d'acteurs spécialisés dans un domaine du circuit de la cueillette à l'écoulement du produit. Mais les limites entre ces différentes catégories de commerçants (les cueilleurs, les «bana bana », les boutiquiers) sont minces voire inexistantes. Chaque acteur peut jouer différents rôles suivant les opportunités, c'est à dire être cueilleur et détaillant. Les acheteurs des produits forestiers aux collecteurs sont en majorité des allochtones qui ne peuvent pas accéder directement à ces produits.

\section{Les prix de commercialisation}

La vaste majorité des PFNL identifiés dans la zone du Kasa est commercialisée sous forme brute. Par exemple les fruits de Saba senegalensis sont très bien consommés et constituent une importante filière commerciale au Sénégal mais aussi en Côte-d'Ivoire et au Burkina Faso (Belem et al., 2008). Les fruits sont les organes les plus commercialisés tel que c'est le cas pour Synsepalum dulcificum au bénin (Fandohan et al. 2017). Seuls quelques produits sont transformés et vendus localement ou dans les capitales régionales. Il s'agit principalement des fruits de Elaeis guinneensis qui donnent l'huile de palme qui est vendue localement et dans tout le pays.

La commercialisation des produits forestiers se fait principalement au marché communal d'Oussouye et au centre touristique de Cap Skirring. Les prix de vente d'un produit varient d'une localité à l'autre. Cette variation des prix est conditionnée par trois critères : l'offre et la demande des marchés, la disponibilité des collecteurs et la facilité de conservation des produits. Ces 
trois paramètres sont pris en compte lors de la détermination de la valeur marchande des produits. En période d'abondance des produits rapidement périssables comme Saba senegalensis et Landolphia heudelotii, les prix chutent considérablement dans les marchés. Par compte, les produits faciles à conserver comme Dialium guineense, Adansonia digitata, Tamarindus indica, Parinari excelsa, Parkia biglobosa, Xylopia aethiopica, la sève fermentée (vin de palme) et l'huile de palme de Elaeis guineensis sont vendus sur les marchés à bons prix (N'Diaye et $a l$., 2002). De même, les fruits très rares tels que ceux de Dialium guineense et de Xylopia aethiopica sont acquis après commande.

\section{Conclusion}

Le présent travail a permis de connaître la diversité des espèces fruitières végétales sauvages comestibles de la zone Kasa et leur contribution dans l'économie locale. L'étude montre 62 espèces exploitées réparties dans 31 familles et 54 genres. Les produits tirés de ces espèces font 1'objet de différentes utilisations regroupées en six catégories. La proportion d'espèces dont les produits font l'objet d'une commercialisation est faible par rapport à la diversité des espèces comestibles recensées. Pour la population enquêtée, ces produits connaissent un regain d'intérêt économique impliquant plusieurs acteurs. Même si la population reconnaît l'exploitation de plusieurs organes pour des besoins divers, dans ce secteur de la commercialisation, c'est le fruit qui est plus prisé. Il est souvent vendu à l'état brut à des prix variables souvent influencés par différents facteurs liés à l'enclavement de la zone de cueillette, à la période de production et à la capacité de conservation du produit. Le marché de ces produits est dominé par les femmes créant ainsi des emplois dans ce secteur. Ces commerçants surtout les «bana bana » jouent un rôle important de régulateur économique dans la commercialisation des produits forestiers. L'apport financier qu'ils procurent aux paysans-collecteurs permet à ces derniers d'apprécier le commerce des produits forestiers et par conséquent de s'investir dans la protection et la valorisation des ressources naturelles forestières. Ces produits sont en grande partie vendus sur place et seule une petite quantité est transitée par les «bana bana et autres commerçants étrangers. De ce fait, on peut retenir que la commercialisation de ces produits forestiers reste donc une activité confinée en milieu rural même si l'on reconnait le développement des marchés locaux potentiels pourvoyeurs d'emplois pour les autochtones. Etant donné que le marché des espèces fruitières comestibles met en jeux plusieurs acteurs, il est nécessaire de pousser la réflexion pour une meilleure organisation des prélèvements et de la commercialisation des produits forestiers. 


\section{Remerciements}

Nous sommes reconnaissants aux chefs des villages, aux guides et surtout aux villageois pour leur entière collaboration dans le recueil des données.

\section{References:}

1. Aboubakar, D., Sorto M, Mbayabe, L., Woin, N., Bourou, S. \& Gandebe, M. (2009). Commercialisation des fruits dans les savanes d'Afrique centrale. Communication au Colloque « Savane Africaines en développement : innover pour durer », 20-24 avril 2009, Garoua, Cameroun. Livre de résumé de la communication. 58 p.

2. Adam, JG (1970). Noms vernaculaires des plantes du Sénégal. Journal d'Agriculture tropicale et de botanique appliquée, T. 17 (7-8-9), 112 p.

3. Agbahoungba, S., Assogbadjo AE, Chadare, FJ, Idohou, R., Salako, VK, Agoyi, EE \& KakaïR, LG (2016). Ecological diversity and conservation of wild edible fruit trees species in the Lama Forest Reserve in Benin. Bois et Forêts des Tropiques, 3 (329), 53-65.

4. Agbankpé, AJ, Dougnon, TV, Bankolé, HS, Yèhouénou, B., Yédomonhan, H., Lègonou, M. \& Dougnon, TJ (2014). Etude ethnobotanique des légumes feuilles thérapeutiques utilisés dans le traitement des diarrhées au sud-Bénin (Afrique de l'Ouest). International Journal of Biological and Chemical Sciences, 8 (4), 1784-1795.

5. Alira, A. (2004). Commercialisation de fruits sauvages et de leurs produits dans Boucle du Mouhoun. Cas des villes de Bissandérou, Bomborokuo, Soana et de la ville de Dédougou. Mémoire. Université Polytechnique de Bobo-Dioulasso. $111 \mathrm{p}$.

6. ANSD (Agence Nationale de la Statistique et de la Démographie) (2015). Situation économique et sociale régionale 2013. Service Régional de la Statistique et de la Démographie de Ziguinchor. Sénégal. 126 p.

7. Apema, R., Mozouloua, D. \& Madiapevo, SN (2010). Inventaire préliminaire des fruits sauvages comestibles vendus sur les marches de Bangui. Dans X. van der Burgt, J. van der Maesen, J.-M. Onana. (éds.). Systématique et conservation des plantes africaines (313-319). Kiew, Royal Botanical Garden, Royaume-Uni.

8. Arbonnier, M. (2002). Arbres, arbuste et lianes des zones sèches d'Afrique de l'Ouest. Deuxième édition, CIRAD, MNHN-UICN, Paris. $541 \mathrm{p}$.

9. Atato, A., Wala, K., Batawila, K., Woegan, AY \& Akpagan, K. (2010). Diversité des fruitiers ligneux spontanés du Togo. Fruit Vegetable Cereal Science and Biotechnol, 4 (1), 1-9. 
10. Baumer, M. (1995). Arbres, arbustes et arbrisseaux nourriciers en Afrique occidentale. Dakar : Enda Tiers-Monde. 260 p.

11. Begossi, A. (1996). Use of ecological methods in ethnobotany: Diversity indices. Economic Botany, 50 (3). 280 p.

12. Belem, B., Smith Olsen, C., Theilade, I., Bellefontaine, R., Guinko, S., Mette Lykke, A., Diallo, A. \& Boussim, JI (2008). Identification des arbres hors forêt préférés des populations du Sanmatenga (Burkina Faso). Bois Forêt des Tropiques, 4 (298), 53-60.

13. Berhaut, J. (1967). Flore du Sénégal plus complète avec les forêts humides de la Casamance. Ed. Claire Afrique, Dakar. 485 p.

14. Berhaut, J. (1971). Flore illustrée du Sénégal.Dicotylédones. Tome 1 Acanthacées à Avicenniacées. Gouvernement du Sénégal-Ministère du développement Rural et de l'Hydraulique, Direction des Eaux et Forêts, Dakar. 626 p.

15. Berhaut, J. (1974). Flore illustrée du Sénégal. Dicotylédones. Tome 2 Balanophoracées à Composées. Gouvernement du Sénégal-Ministère du développement Rural et de l'Hydraulique, Direction des Eaux et Forêts, Dakar. 695 p.

16. Berhaut, J. (1975a). Flore illustrée du Sénégal. Dicotylédones. Tome 3 Connaracées à Euphorbiacées. Gouvernement du Sénégal-Ministère du développement Rural et de l'Hydraulique, Direction des Eaux et Forêts, Dakar. 634 p.

17. Berhaut, J. (1975b). Flore illustrée du Sénégal. Dicotylédones. Tome 4 Ficoidées à Légumineuses. Gouvernement du Sénégal-Ministère du développement Rural et de 1'Hydraulique, Direction des Eaux et Forêts, Dakar. 625 p.

18. Berhaut, J. (1976). Flore illustrée du Sénégal. Dicotylédones. Tome 5 Légumineuses Papilionacées. Gouvernement du Sénégal-Ministère du développement Rural et de 1'Hydraulique, Direction des Eaux et Forêts, Dakar. 658 p. conservational significance. Oikos63: 19-23.

19. Berhaut, J. (1979). Flore illustrée du Sénégal. Dicotylédones. Tome 6 Linaceae à Nyphéaceae. Gouvernement du Sénégal-Ministère du développement Rural et de 1'Hydraulique, Direction des Eaux et Forêts, Dakar. 636 p.

20. Berhaut, J. (1995). Nouvelle Flore illustrée du Sénégal. Monocotylédones. Tome 9 et 10. Gouvernement du Sénégal-Ministère du développement Rural et de l'Hydraulique, Direction des Eaux et Forêts, Dakar. 788 p.

21. Betti, JL, Ngankoué, CM, Dibong, SD \& Singa, AE (2016). Etude ethnobotanique des plantes alimentaires spontanées vendues dans les marchés de Yaoundé, Cameroun. International Journal of Biological and Chemical Sciences, 10 (4), 1678-1693. 
22. Bikoué, MAC \& Essomba, H. (2007). Gestion des ressources naturelles fournissant les PFNL alimentaires en Afrique centrale. Document de Travail $\mathrm{N}^{\circ} 5$. Organisation des Nations Unies pour l'Alimentation et l'Agriculture. $104 \mathrm{p}$.

23. Boukari, I., Shier, NW, Frisch, J., Watkins, BA, Pawloski, L. \& Fly, AD (2001). Calcium analysis of selected western African foods. Journal of Food Composition and Analysis, 14 (1), 37 - 42.

24. Bourou, S., Ndiaye, F., Diouf, M., Diop, T. \& Van Damme, P. (2010). Tamarind (Tamarindus indica L.) parkland mycorrhizal potential within three agroecological zones of Senegal. Fruits, 65 (6), 377-385.

25. Core Team, R. (2017). $R$ : A language and environment for statistical computing. $\mathrm{R}$ Foundation for Statistical Computing, Vienna, Austria.URL https://www.R-project.org/.)

26. Dibong, SD, Emmanuel, MM \& Ngoye, A. (2011). Vulnérabilité des espèces à fruits sauvages vendus dans les marchés de Douala (Cameroun). Journal of Animal \& Plant Sciences, 11 (3), 1435-1441.

27. Dieng, SD, Diop, M., Faye, LC, Goudiaby, A., Niang-Diop, F., Guiro, I., Sambou, S., Lykke, AM \& Sambou, B. (2016b). Characterization of Ecosystem Services Provided by Detarium microcarpum around the Protected Forest of Patako (Senegal). Journal of Applied Environmental and Biological Sciences, 6 (12), 41-49.

28. Dieng, SD (2017). Evaluation des services écosystémiques fournis par Cordyla pinnata (Lepr. Ex A. Rich.) Milne-Redh., Detarium microcarpum Guill. Et Perr. et Detarium senegalense (J.F. Gmel.) : cas de la Forêt Classée de Patako et de ses environs (Centre-Ouest du Sénégal). Thèse de doctorat en Sciences de l'Environnement, Faculté des Sciences et Techniques, Université Cheikh Anta Diop de Dakar. $184 \mathrm{p}$.

29. Dieng, SD, Diop, M., Goudiaby, A., Niang-Diop, F., Faye, LC, Guiro, I., Sambou, S., Lykke, AM \& Sambou, B. (2016a). Caractérisation des services écosystémiques fournis par Cordyla pinnata dans la périphérie de la Forêt classée de Patako au Sénégal ». VertigO - la revue électronique en sciences de l'environnement [En ligne], 16 (2), 1-17.

30. Diop, M. (2011). L'arbre et la Forêt. Usages, préférences, représentations et croyances chez les populations riveraines de la

Forêt Classée de Patako (région de Fatick, Sénégal). Thèse Environnement, UCAD. 201 p.

31. El-Siddig, K, Gunasena, HPM, Prasad, BA, Pushpakumara, DKNG, Ramana, KVR, Vijayand, P. \& Williams, JT (2006). Tamarind (Tamarindus indica L.), Br. Libr., Southampt. U.K. 126 p.

32. Fandohan, AB, Chadare FJ, Gouwakinnou GN, Tovissode CF, Bonou A., Djonlonkou, SFB, Houndelo, LFH, Sinsin, CLB \& Assogbadjo, 
AE (2017). Usages traditionnels et valeur économique de Synsepalum dulcificum au Sud-Bénin. Bois et Forêts des Tropiques, 332 (2), 1730.

33. Hawthorne, W. \& Jongkind, C. (2006). Woody plants of western African forests: a guide to the forest trees, shrubs and lianes from Senegal to Ghana. Ed. Royal Botanic Gardens, Kew.

34. Hutchinson, J. \& Dalziel, JM (1954). Flora of West Tropical Africa. 2nd ed. Revised by R. W.J. Keay. et al., Crown Agents for Oversea Governments and Administrations. London; Vol. I. part I. 295 p.

35. Kouebou, C., Goygoy, F., Bourou, S., Djakissam, PK, Layla, H., Zenabou, G., Barbi, M., Vunyingah, M. \& Woin, N. (2013). Biodiversité et valeur alimentaire des fruits au Cameroun: observations préliminaires dans le Département de la Bénoué (Région du Nord). Journal of Applied Biosciences, 69, 5523-5533.

36. Kouyate, AM, Meyer, A., Van Damme, P. \& Diawara, H. (2009). Usages magico-médicinaux et vétérinaires de Detarium microcarpum (Fabaceae) au sud du Mali. dans X. van der Burgt, J. van der Maesen \& J.-M. Onana (eds), Systematics and Conservation of African Plants (367-374). Royal Botanic Gardens, Kew.

37. Lebrun, JP \& Stork, AL (1991, 1992, 1995, 1997). Enumération des plantes à fleurs d'Afrique tropicale. Genève : Conservatoire et Jardin botanique de Genève, 1-4.

38. Malela, KE, Miabangana, ES, Petit, J., N'zikou, JM \& Scher, J. (2016). Enquête ethnobotanique sur les fruits comestibles de la flore spontanée de la République du Congo. Int. J. Pure App. Biosci., 4 (2), 346 - 357.

39. Mangambu, MJDD, Aluma, KJY, Ruurd, VD, Rugenda-Banga, RADD, Mushangalusa, KF, Chibembe, SA, Ntahobavuka, HH, Radar, NB \& Robbrecht, E., 2015. Etude ethnobotanique et ethnolinguistique des ressources forestières ligneuses utilisées par la population du couloir écologique du parc national de Kahuzi-Biéga (R.D. congo). European Scientific Journal, 11 (15), 135-162.

40. Martin, GJ (1995). Ethnobotany. A method manual. London: Chapman \& Hall. 268 p.

41. Mbaye, E. (2006). La cueillette en brousse : terroirs et filières $d u$ Gandoul. Fonctionnement, logiques, perspectives.... Thèse. Département de géographie, Université Cheikh Anta Diop de Dakar. $404 \mathrm{p}$.

42. Mbula Itumba, O., (2014). Contribution à l'étude des plantes alimentaires sauvages de Yasikia (PK 31 route OPALA, PO, RDC). Universite de kisangani, RDC. 36 p. 
43. N'Diaye, M., Kéita, FB \& Martin, P. (2002). Principaux fruits de cueillette consommés et commercialisés en Guinée. Fruit, 58 (2), 99116.

44. Ndiaye, I., Camara, B., Ngom, D., \& Sarr, O. (2017). Diversité spécifique et usages ethnobotaniques des ligneux suivant un gradient pluviométrique Nord-Sud dans le bassin arachidier sénégalais. Journal of Applied Biosciences, 113, 11123-11137.

45. Ouattara, ND, Gaille, E., Stauffer, FW \& Bakayoko, A. (2016). Diversité floristique et ethnobotanique des plantes sauvages comestibles dans le Département de Bondoukou (Nord- Est de la Côte d'Ivoire). Journal of Applied Biosciences, 98, $9284-9300$.

46. Sahoré, AS, Nemlin, JG \& Tetchi, AF (2012). Study of physicochemical properties of some traditional vegetables in Ivory Coast: Seeds of Beilschmiedia mannii (Lauraceae), Seeds of Irvingia gabonensis (Irvingiaceae) and Volvariella volvaceae. Food and Nutrition Sciences. 3 (1), 14-17.

47. Sambou, B. (2004). Evaluation de l'Etat, de la dynamique et des tendances évolutives de la flore et de la végétation ligneuses dans les domaines soudanien et sub-guinéen au Sénégal. Thèse de Doctorat d'Etat ès Sciences Naturelles, Université Cheikh AntaDiop de Dakar, Sénégal, $210 \mathrm{p}$.

48. Trotter, RT \& Logan, MH (1986). Informant Consensus: a New Approach for Identifying Potentially Effective Médicinal Plants. Edn. Bedfore Hills: New York, 91-112. 


\section{Annexe}

Tableau 2 : Liste des espèces végétales utilisées (en abrégé) pour AFC

\begin{tabular}{|c|c|c|}
\hline Allophyllus africanus (A-afri) & Drypetes floribunda (D-flori) & Passiflora foetida (P-foeti) \\
\hline Atroxima afzeliana (A-afze) & Dialium guineense (D-guine) & $\begin{array}{l}\text { Pseudospondias microcarpa (P- } \\
\text { micro) }\end{array}$ \\
\hline Aframomum cereum (A-cer) & Detarium senegalense (D-sene) & Psychotria peduncularis (P-pedun) \\
\hline Adansonia digitata (A-digi) & Elaeis guineensis (E-guine) & Phoenix reclinata (P-recli) \\
\hline Aframomum elliotii (A-ellio) & Ficus capensis (F-cap) & Ritchiea capparoides (R-cappa) \\
\hline Avicennia germinans (A-ger) & Ficus lutea (F-lutea) & Synsepalum brevipes (S-brevi) \\
\hline Annona glauca (A-glau) & Gardenia erubescens (G-eru) & Sorindeia juglandifolia (S-juglan) \\
\hline Azadirachta indica (A-Indi) & Icacina oliviformis (I-olivi) & Sarcocephalus latifolius (S-lati) \\
\hline Anacardium occidentale (A-occi) & Landolphia dulcis (L-dul) & Spondias mombin (S-mom) \\
\hline Annona senegalensis (An-sene) & heudelotii $(\mathrm{L}-$ & Saba senegalensis (Sab-sene) \\
\hline Aphania senegalensis (Ap-sene) & Landolphia hirsuta (L-hirsu) & Salacia senegalensis (Sal-sene) \\
\hline Balanites aegyptiaca (B-aegyp) & Landolphia owariensis (L-owa) & Treculia africana (T-afri) \\
\hline Borassus aethiopum (B-aethio) & Mammea africana (M-afri) & Tamarindus indica (T-indi) \\
\hline Cola cordifolia (C-cordi) & Monanthotaxis barteri (M-bar) & Uvaria chamae (U-chamae) \\
\hline Calamus deerratus (C-deer) & Mangifera indica (M-indi) & Uvaria thomasii (U-thoma) \\
\hline Chrysobalanus ellipticus (C-ellip) & $\begin{array}{l}\text { Macrosphyra longistyla (M- } \\
\text { longis) }\end{array}$ & Voacanga africana (V-afri) \\
\hline Cucumis metuliferus (C-metu) & $\begin{array}{l}\text { Neocarya macrophylla }(\mathrm{N}- \\
\text { macro) }\end{array}$ & Vitex doniana (V-donia) \\
\hline $\begin{array}{l}\text { Chrysobalanus orbicularis (C- } \\
\text { orbi) }\end{array}$ & Nymphaea-spp & Xylopia aethiopica (X-aethio) \\
\hline Ceiba pentandra (C-pentan) & Parkia biglobosa (P-biglo) & Ziziphus mauritiana (Z-mauri) \\
\hline Celtis toka (C-toka) & Pancovia bijuga (P-bijuga) & $\begin{array}{l}\begin{array}{l}\text { Zanthoxylum zanthoxyloides (Z- } \\
\text { zantho) }\end{array} \\
\end{array}$ \\
\hline Diospyros ferrea (D-fer) & Parinari excelsa (P-excel) & \\
\hline
\end{tabular}

